

\title{
Diboson Results from CMS
}

\section{Nate Woods*}

University of Wisconsin-Madison (US)

E-mail: nwoods@hep.wisc.edu

\section{On behalf of the CMS Collaboration}

Measurements of diboson production in proton-proton collisions at the LHC, performed by the CMS Collaboration, are summarized. We report studies of ZZ production at $\sqrt{s}=13 \mathrm{TeV}, \mathrm{WZ}$ production at $\sqrt{s}=8$ and $13 \mathrm{TeV}, \mathrm{WV} \rightarrow \ell v \mathrm{q} \overline{\mathrm{q}}\left(\mathrm{V}=\mathrm{W}^{ \pm}\right.$or $\mathrm{Z}, \ell=\mathrm{e}$ or $\left.\mu\right)$ at $\sqrt{s}=8$ and $13 \mathrm{TeV}$, and $\mathrm{Z} \gamma \rightarrow v \bar{v} \gamma$ at $\sqrt{s}=13 \mathrm{TeV}$. Inclusive and differential cross section measurements and limits on anomalous triple gauge couplings are reported, as well as a measurement of the $\mathrm{Z} \rightarrow 4 \ell$ branching fraction.

The European Physical Society Conference on High Energy Physics 5-12 July

Venice, Italy

${ }^{*}$ Speaker. 


\section{Introduction}

Measurements of diboson production ( $\mathrm{VV}$, where $\mathrm{V}$ is $\mathrm{Z}, \mathrm{W}^{ \pm}$, or $\gamma$ ) at the CERN LHC can provide insights into the electroweak gauge structure of the standard model (SM). With a large, exhaustively studied dataset at $\sqrt{s}=8 \mathrm{TeV}$ from 2012, a dataset at the unprecedented center-ofmass energy of $13 \mathrm{TeV}$ from 2015, and an exceptionally large dataset at $\sqrt{s}=13 \mathrm{TeV}$ from 2016, the CMS experiment can study these processes at new energies and in great detail. In this report, we briefly summarize measurements of $\mathrm{ZZ}$ [1], WZ [2, 3], WV $\rightarrow \ell v \mathrm{q} \overline{\mathrm{q}}[4,5]$ (where $\mathrm{V}$ may be $\mathrm{W}^{ \pm}$or $\mathrm{Z}$ and $\ell$ may be e or $\mu$ ), and $\mathrm{Z} \gamma \rightarrow \nu \bar{v} \gamma[6]$ production. Full analysis details and results can be found in these references.

\section{ZZ Production}

ZZ production provides a probe of neutral gauge boson interactions in the SM. The cross section is small but highly sensitive to higher-order QCD corrections, which have recently been calculated to next-to-next-to-leading order (NNLO) [7]. The $\ell^{+} \ell^{-} \ell^{\prime+} \ell^{\prime-}\left(\ell, \ell^{\prime} \in \mathrm{e}, \mu\right)$ channel provides a fully reconstructible final state with small backgrounds. The CMS measurement of fourlepton production [1], performed on the 2016 dataset with an integrated luminosity of $35.9 \mathrm{fb}^{-1}$ at $\sqrt{s}=13 \mathrm{TeV}$, therefore uses relaxed lepton identification and isolation criteria without admitting large $\mathrm{Z}+$ jets backgrounds. The remaining small $\mathrm{Z}+$ jets and $\mathrm{t} \overline{\mathrm{t}}$ contributions are estimated from $Z+\ell^{\prime} \ell^{\prime}$ control samples where one or both $\ell^{\prime}$ fail the analysis selection criteria using a per-lepton "loose-to-tight" transfer factor obtained from a $\mathrm{Z}+\ell_{\text {fake }}$ sample. Triboson, $\mathrm{t} \mathrm{Z} Z$, and Higgs boson backgrounds are estimated with simulated samples. Both $\mathrm{Z}$ boson candidates in each event are required to have invariant mass in the range $60<m_{\ell \ell}<120 \mathrm{GeV}$. The four-lepton invariant mass distribution is shown in Fig. 1 (left), along with SM predictions with which it generally agrees. The total cross section for events with two $\mathrm{Z}$ bosons with mass in the range $60-120 \mathrm{GeV}$ is calculated from these data and found to be

$$
\sigma(\mathrm{pp} \rightarrow \mathrm{ZZ})=17.8 \pm 0.6(\text { stat })_{-0.6}^{+0.7}(\text { syst }) \pm 0.4(\text { theo }) \pm 0.5 \text { (lumi) } \mathrm{pb}
$$

which may be compared to the NNLO prediction of $16.2_{-0.4}^{+0.6} \mathrm{pb}$ from MATRIX [7]. CMS and ATLAS total ZZ cross section measurements are shown as a function of $\sqrt{s}$ along with NLO and NNLO theory predictions in Fig. 1 (right). Differential cross sections were calculated as functions of a number of kinematic observables and can be seen in Ref. [1].

Limits on anomalous triple gauge couplings (aTGC) are set in the effective Lagrangian framework [8]. Example aTGC signal hypotheses are shown compared to data in Fig. 1 (left). Limits are set at $95 \%$ confidence level separately for all four fully neutral aTGC parameters,

$$
\begin{aligned}
& -0.0012<f_{4}^{Z}<0.0011, \quad-0.0010<f_{5}^{Z}<0.0013, \\
& -0.0013<f_{4}^{\gamma}<0.0013, \quad-0.0012<f_{5}^{\gamma}<0.0013,
\end{aligned}
$$

the most stringent set to date on these parameters. Two-dimensional limits were also set, and can be seen in Ref. [1]. 

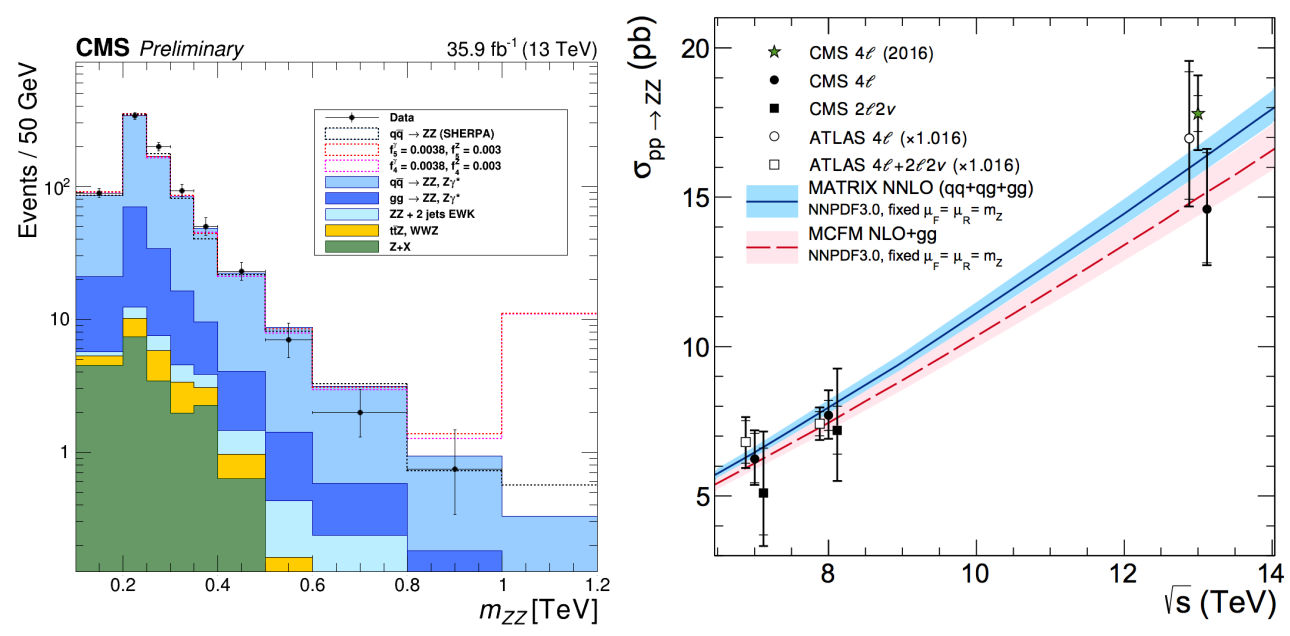

Figure 1: (Left): Distribution of the four-lepton invariant mass $m_{\mathrm{ZZ}}$ of all events with $60<m_{\mathrm{Z}_{1}, \mathrm{Z}_{2}}<$ $120 \mathrm{GeV}$. Points represent data, with statistical uncertainty bars. The stack of filled histograms represents the SM signal prediction and background estimate, with a grey band showing the sum in quadrature of the statistical and systematic uncertainties on the total expected yield. Dotted lines represent two potential aTGC models. (Right): The total $\mathrm{pp} \rightarrow \mathrm{ZZ}$ cross section as a function of $\sqrt{s}$ from CMS and ATLAS, compared to the NLO prediction from MCFM and the NNLO prediction from MATRIX. For details of the calculations, see Ref. [1].

\section{1 $\mathrm{Z} \rightarrow 4 \ell$ Branching Fraction}

Widening the allowed dilepton mass range to $40<m_{\ell \ell}<120 \mathrm{GeV}, 4<m_{\ell^{\prime} \ell^{\prime}}<120 \mathrm{GeV}$ expands the analysis to include nonresonant $\mathrm{Z} \gamma^{*}$ and $\gamma^{*} \gamma^{*}$ production, and the Higgs and $\mathrm{Z}$ boson resonances. The $\mathrm{Z} \rightarrow \ell \ell \gamma^{*} \rightarrow 4 \ell$ branching fraction is measured for events with $80<m_{4 \ell}<100 \mathrm{GeV}$ by comparing the measured $\mathrm{Z} \rightarrow 4 \ell$ fiducial cross section to the $\mathrm{Z} \rightarrow 2 \ell$ fiducial cross section, with a correction for nonresonant four-lepton production, and is found to be

$$
\mathscr{B}(\mathrm{Z} \rightarrow 4 \ell)=4.8 \pm 0.2 \text { (stat) } \pm 0.2 \text { (syst) } \pm 0.1 \text { (theo) } \pm 0.1 \text { (lumi) } \times 10^{-6},
$$

which agrees with the theoretical prediction of $4.6 \times 10^{-6}$.

\section{WZ Production}

Like ZZ, WZ production is sensitive to higher-order QCD corrections and the details of trilinear gauge boson couplings, and the $3 \ell v$ final state provides a clean channel with manageable backgrounds. The signature of the process is three well-identified, isolated leptons in association with a large imbalance of transverse momentum of detected particles (the magnitude of which is called the missing energy, $E_{\mathrm{T}}^{\text {miss }}$ ) left by the undetected neutrino. In CMS WZ measurements, the $\mathrm{Z}$ boson must have invariant mass $76<m_{\ell \ell}<106 \mathrm{GeV}$, and the trilepton invariant mass must exceed $100 \mathrm{GeV}$. Background estimation is performed by extrapolating the nonprompt contributions to the signal region from control regions with one, two, or all three leptons failing the ID or isolation criteria, via per-lepton transfer factors calculated in a dijet sample. The inclusive WZ cross section 
was measured with the 2015 dataset $\left(2.3 \mathrm{fb}^{-1}\right.$ at $\left.\sqrt{s}=13 \mathrm{TeV}\right)$ [3], and found to be

$$
\sigma(\mathrm{pp} \rightarrow \mathrm{WZ})=39.9 \pm 3.2(\text { stat })_{-3.1}^{+2.9}(\text { syst }) \pm 0.4(\text { theo }) \pm 1.3 \text { (lumi) } \mathrm{pb}
$$

which is compatible with the NLO prediction of $46.1_{-3.9 \%}^{+4.9 \%}$ and in slight tension with the NNLO prediction of $51.1_{-2.0 \%}^{+2.2 \%}$ from MATRIX [9]. The total WV cross section is shown in Fig. 2 (left). Differential WZ fiducial cross sections, seen as a function of $\mathrm{Z}$ boson $p_{\mathrm{T}}$ in Fig. 2 (right), and limits on charged aTGC parameters, were calculated with the 2012 dataset, which corresponds to $19.6 \mathrm{fb}^{-1}$ at $\sqrt{s}=8 \mathrm{TeV}$, and can be found in Ref. [2]. Differential cross sections were unfolded with five iterations of the method described in Ref. [10].
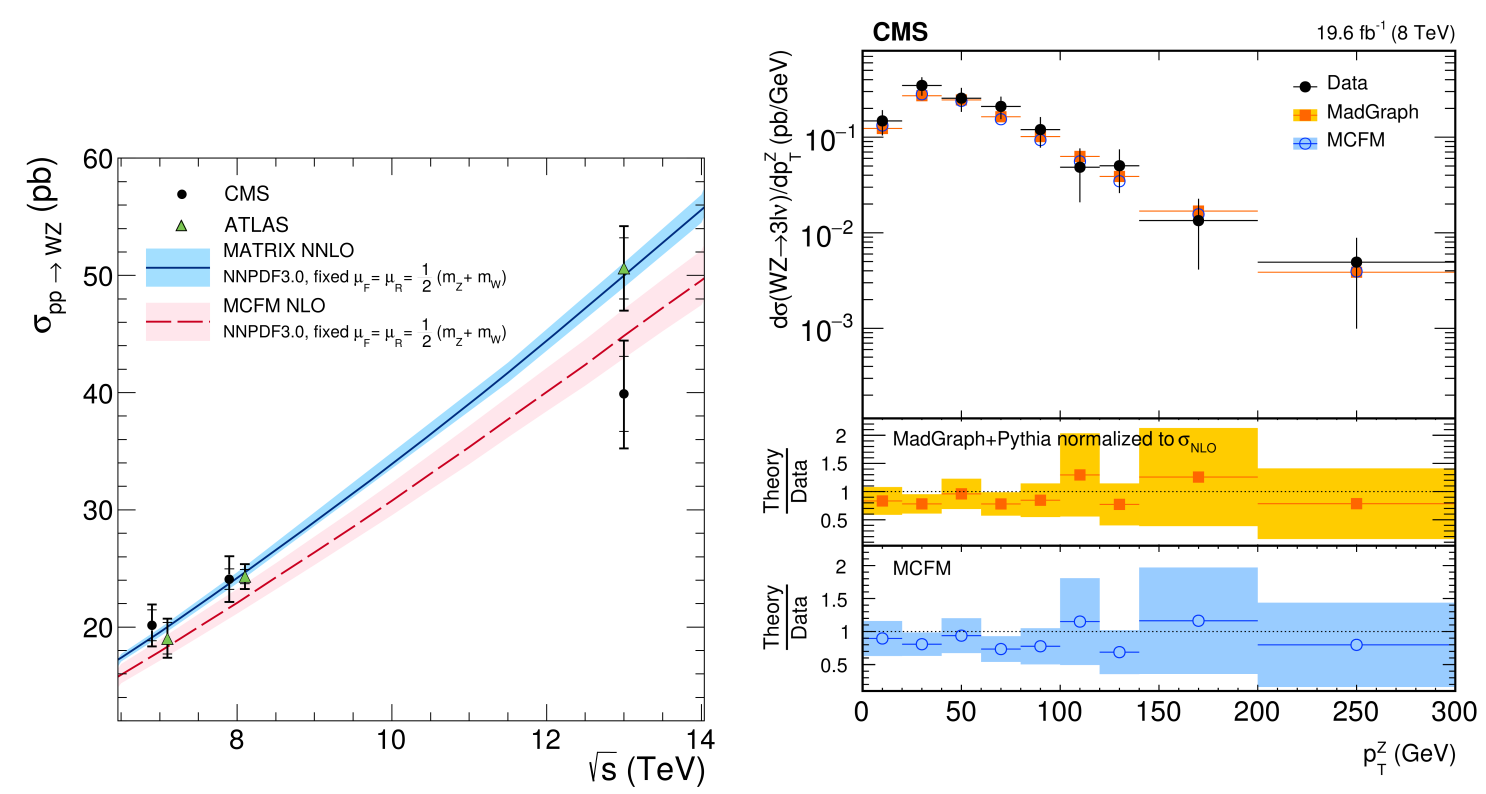

Figure 2: (Left): The total $\mathrm{pp} \rightarrow \mathrm{WZ}$ cross section as a function of $\sqrt{s}$ from CMS and ATLAS, compared to the NLO prediction from MCFM and the NNLO prediction from MATRIX. For details of the calculations, see Ref. [3]. (Right): The WZ $\rightarrow 3 \ell v$ differential cross section as a function of $Z$ boson $p_{\mathrm{T}}$. Solid circles represent data, open circles represent the NLO prediction from MCFM, and squares represent the LO prediction from MADGRAPH. For details of the calculation, see Ref. [2].

\section{4. $\mathbf{W V} \rightarrow \ell v \mathbf{q} \overline{\mathbf{q}}$}

Diboson events in which one boson decays to $q \bar{q}$ are attractive because the $\mathrm{V} \rightarrow \mathrm{q} \overline{\mathrm{q}}$ branching fractions are substantially higher than the leptonic branching fractions. Even with the other boson's leptonic decay to provide a measure of background discrimination, $\mathrm{V}+$ jets rates at $\mathrm{LHC}$ are too high for cross section measurements with hadronic channels. If the hadronically decaying boson has a high transverse boost, the two quark jets merge into a single "fat" jet which may be differentiated from QCD jets with substructure techniques such as pruning [11] and N-subjettiness [12]. This boosted regime corresponds to events with high invariant mass, which are sensitive many new physics models, so aTGC searches were performed in the WV $\rightarrow \ell v \mathrm{q} \overline{\mathrm{q}}$ channel with the 2012 [4] 
and 2015 [5] datasets. The leptonic $\mathrm{W}$ is constructed from a lepton and $E_{\mathrm{T}}^{\mathrm{miss}}$, as before, but it is required to have $p_{\mathrm{T}}>200 \mathrm{GeV}$. The hadronic boson is constructed from a fat jet, which must also have $p_{\mathrm{T}}>200 \mathrm{GeV}$, and is identified as an electroweak gauge boson by its substructure. Backgrounds from $\mathrm{W}+$ jets and $t \bar{t}$ are suppressed with a veto on extra jets in the event and by topological requirements. Remaining backgrounds are modeled by taking their shape from simulated samples and obtaining the normalization for each process with simultaneous shape fits on the fat jet pruned mass distribution. Limits on charged aTGC parameters in the effective Lagrangian formalism [8] are extracted with shape fits to the jet $p_{\mathrm{T}}$ distribution (2012 data) or the WV invariant mass distribution above $900 \mathrm{GeV}$ (2015 data), both of which are shown in Fig. 3 for $\mu v \mathrm{q} \overline{\mathrm{q}}$ events. The $95 \%$ CL limits set with the 2012 data,

$$
-0.011<\lambda_{\mathrm{Z}}<0.011, \quad-0.044<\Delta \kappa_{\gamma}<0.063, \quad-0.0087<\Delta g_{1}^{\mathrm{Z}}<0.024,
$$

are the most stringent to date on $\Delta \kappa_{\gamma}$ and $\Delta g_{1}^{Z}$. Two-dimensional limits are also set and can be seen in Refs. [4, 5].
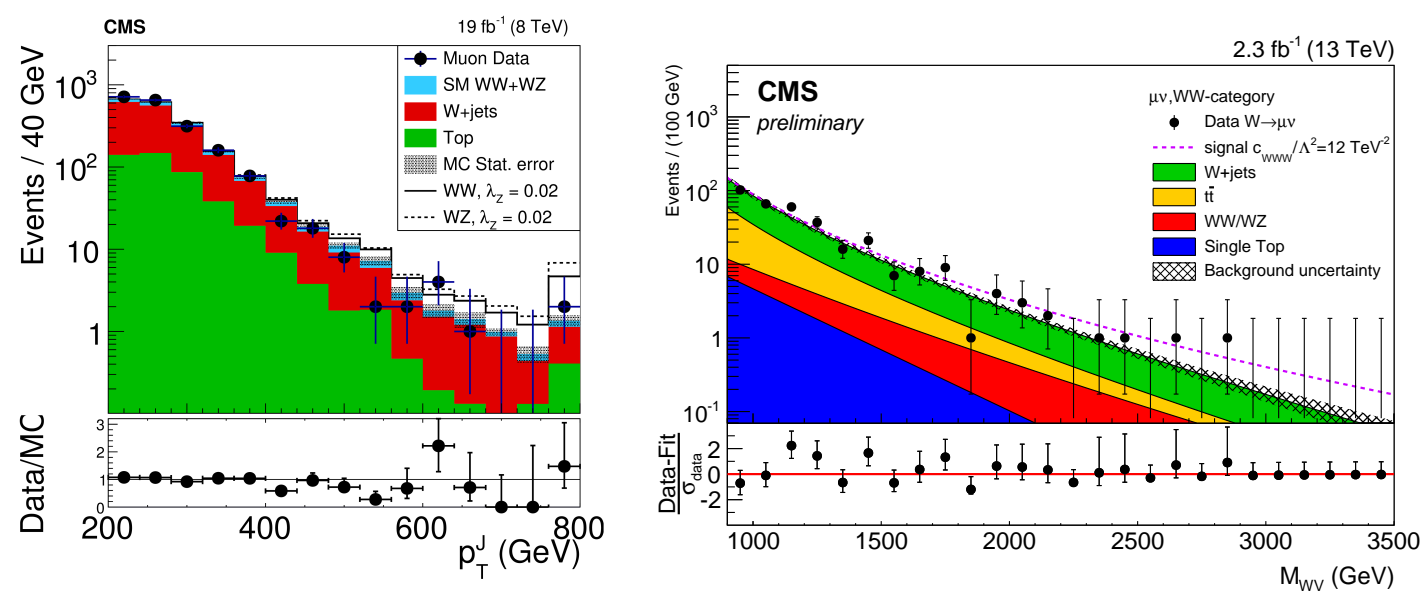

Figure 3: (Left): Hadronic $\mathrm{V} p_{\mathrm{T}}$ in $\mathrm{pp} \rightarrow \mathrm{WV} \rightarrow \mu v \mathrm{q} \overline{\mathrm{q}}$ events at $\sqrt{s}=8 \mathrm{TeV}$ [4]. (Right): Invariant mass of the WV system in pp $\rightarrow \mathrm{WV} \rightarrow \mu v \mathrm{q} \overline{\mathrm{q}}$ events at $\sqrt{s}=13 \mathrm{TeV}$ [5]. In both, points represent data, solid histograms represent the SM prediction, and dotted curves represent the predictions of potential aTGC hypotheses.

\section{5. $\mathbf{Z} \gamma \rightarrow v \bar{v} \gamma$}

In the $\mathrm{SM}$, the $\mathrm{pp} \rightarrow \mathrm{Z} \gamma \rightarrow v \bar{v} \gamma$ production process occurs only through initial state photon radiation, and is therefore sensitive to anomalous $\mathrm{Z} \gamma$ couplings. It is also a primary background process for dark matter searches in the $\gamma+E_{\mathrm{T}}^{\text {miss }}$ channel. The fiducial cross section for this process has been measured with the 2015 dataset for single photon events with $E_{\mathrm{T}}^{\gamma}>175 \mathrm{GeV}$ and $\left|\eta^{\gamma}\right|<1.44$ [6]. At reconstruction level, each event is required to have $E_{\mathrm{T}}^{\mathrm{miss}}>170 \mathrm{GeV}$. Backgrounds from $\mathrm{W} \gamma \rightarrow \mathrm{e} v \gamma$ events and events where an electron or jet is misidentified as a photon are rejected with a veto on extra leptons and topological requirements. Residual contributions from these processes are estimated from a sample of events in which the requirement that there be no 
seed track in the silicon pixel tracker subdetector pointing to the photon is inverted, using transfer factors derived from the known pixel seed efficiency. Experimental backgrounds from beam halo and light spikes in the electromagnetic calorimeter (ECAL) are rejected with cuts on the ECAL signals' timing and shape, and remaining contributions are estimated with fits to the timing signal shapes. The fiducial cross section is found to be

$$
\sigma_{\text {fid }}(\mathrm{pp} \rightarrow \mathrm{Z} \gamma \rightarrow v \bar{v} \gamma)=66.5 \pm 13.6 \text { (stat) } \pm 14.3 \text { (syst) } \pm 2.2 \text { (lumi) fb }
$$

which is compatible with the NNLO prediction of $65.5 \pm 3.3 \mathrm{fb}$ from MATRIX [13].

\section{References}

[1] CMS Collaboration, Measurement of the $p p \rightarrow Z Z$ production cross section, $Z \rightarrow 4 \ell$ branching fraction and constraints on anomalous triple gauge couplings at $\sqrt{s}=13 \mathrm{TeV}$, CMS-PAS-SMP-16-017, 2017. http://cds.cern.ch/record/2256100.

[2] CMS Collaboration, Measurement of the WZ production cross section in pp collisions at $\sqrt{s}=7$ and $8 \mathrm{TeV}$ and search for anomalous triple gauge couplings at $\sqrt{s}=8 \mathrm{TeV}$, Eur. Phys. J. C 77 (2017) 236, [1609.05721].

[3] CMS Collaboration, Measurement of the WZ production cross section in pp collisions at $\sqrt{s}=13 \mathrm{TeV}$, Phys. Lett. B 766 (2017) 268, [1607.06943].

[4] CMS Collaboration, Search for anomalous couplings in boosted $W W / W Z \rightarrow \ell v q \bar{q}$ production in proton-proton collisions at $\sqrt{s}=8 \mathrm{TeV}$, Phys. Lett. B 772 (2017) 21, [1703.06095].

[5] CMS Collaboration, Search for anomalous couplings in semileptonic WW and WZ decays at $\sqrt{s}=13 \mathrm{TeV}$, CMS-PAS-SMP-16-012, 2016. https://cds.cern.ch/record/2209148.

[6] CMS Collaboration, Measurement of the production cross section for pp to $Z(v v) \gamma$ at $\sqrt{s}=13 \mathrm{TeV}$ at CMS, CMS-PAS-SMP-16-004, CERN, Geneva, 2016. https://cds.cern.ch/record/2204922.

[7] F. Cascioli, T. Gehrmann, M. Grazzini, S. Kallweit, P. Maierhöfer, A. von Manteuffel et al., ZZ production at hadron colliders in NNLO QCD, Phys. Lett. B 735 (2014) 311, [1405. 2219 ].

[8] K. Hagiwara, S. Ishihara, R. Szalapski and D. Zeppenfeld, Low-energy effects of new interactions in the electroweak boson sector, Phys. Rev. D 48 (1993) 2182.

[9] M. Grazzini, S. Kallweit, D. Rathlev and M. Wiesemann, $W^{ \pm} Z$ production at hadron colliders in NNLO QCD, Phys. Lett. B 761 (2016) 179, [1604.08576].

[10] G. D’Agostini, A Multidimensional unfolding method based on Bayes' theorem, Nucl. Instrum. Meth. A 362 (1995) 487.

[11] S. D. Ellis, C. K. Vermilion and J. R. Walsh, Recombination Algorithms and Jet Substructure: Pruning as a Tool for Heavy Particle Searches, Phys. Rev. D 81 (2010) 094023, [0 912 . 0033 ].

[12] J. Thaler and K. Van Tilburg, Identifying Boosted Objects with N-subjettiness, JHEP 03 (2011) 015, [1011.2268].

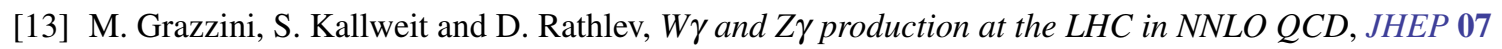
(2015) 085, [1504.01330]. 